\title{
Effects of Drive Amplitude on Continuous Jet Break-up
}

Claire Mcllroy ${ }^{1, \text { a) }}$ and Oliver G. Harlen ${ }^{1, b)}$

Department of Applied Mathematics, University of Leeds, Leeds, LS2 9JT, $U K$

(Dated: 28 May 2019)

We develop a one-dimensional (1D) model of jet break-up in continuous inkjet printing to explore the non-linear behaviour caused by finite-amplitude modulations in the driving velocity, where jet stability deviates from classic (linear) 'Rayleigh' behaviour. At low driving amplitudes and high Weber numbers the spatial instability produces drops that pinch-off downstream of the connecting filament, leading to the production of small satellite droplets between the main drops. On the other hand, we identify a range of driving amplitudes where pinching becomes 'inverted', occurring upstream of the filament connecting the main drops, rather than downstream. This inverted break-up is preferable in printing, as it increases the likelihood of satellite drops merging with the main drops. We find that this behaviour can be controlled by the addition of a second harmonic to the driving signal. This model is in quantitative agreement with a full axisymmetric simulation, which incorporates nozzle geometry.

\section{INTRODUCTION}

Inkjet printing is becoming a powerful manufacturing tool; it is ideal for a wide range of applications due to the advantages of being flexible, non-contact and scalable. In particular, drop-on-demand (DoD) inkjets may be used as robotic pipettes to create micro arrays, fabricate three-dimensional objects or print electrical and optical devices ${ }^{1}$.

a)Electronic mail: claire.mcilroy@nottingham.ac.uk; School of Mathematical Sciences, University of Nottingham, Nottingham, NG7 2

b) Electronic mail: o.g.harlen@leeds.ac.uk
However, while DoD technology is highly versatile, it is currently slow compared to direct printing and continuous inkjet (CIJ) printing. For example, Liu et at study the range of experimental parameters required for stable drop-on-demand inkjet performance.

CIJ provides high-speed printing, but low resolution in the final printed text, thus is ideal for labelling applications. However, CIJ is impractical for more advanced processes requiring high precision. Extending CIJ to more advanced processes requiring high precision requires an improved understanding of the formation of small drops 
from liquid jets, as well as their in-flight behaviour. Many studies have contributed to improving this understanding; for example see Refs. ${ }^{3-7}$. More recently, studies have investigated droplet formation in step emulsifiers $^{8}$ and bifurcating microchannels ${ }^{9}$, and new experimental techniques have been developed to investigate thread breakup ${ }^{10}$.

In CIJ printing, as the name suggests, a continuous jet of liquid is ejected from a large reservoir through a nozzle orifice. Surface tension renders the jet unstable, creating a succession of 'main' drops connected by thin filaments. Depending on the nature of the applied disturbance, these filaments may detach from the main drops at break-up to form smaller 'satellite' drops. The formation of satellite drops during CIJ printing is problematic for a number of reasons. If satellites fail to merge with the main drops, then they may cause splash on the substrate and compromise print quality, or they may migrate towards the nozzle plate as smaller drops are deflected further by electrostatic fields and cross-stream airflows.

Rayleigh $^{11}$ was first to establish that a liquid jet will be rendered unstable by surface tension provided that its wavelength exceeds its circumference. Linear stability analysis of the Navier-Stokes equations leads to a dispersion relation to describe how the growth rate of a disturbance depends on its wave- length. Maximising the dispersion relation reveals that the fastest growing wavelength is approximately 9 times the jet radius for low-viscosity liquids. However, linear theories based on Rayleigh's stability analysis do not predict the formation of satellite drops.

Consequently, weakly non-linear theories have been developed to investigate satellite formation. In particular, both Yeun et al. ${ }^{12}$ and Lee et $a l .{ }^{13}$ use the method of strained coordinates to develop a solution for a symmetric infinite jet, based on a temporal disturbance of the free surface. Qualitatively, satellites appear through two properties of the Newtonian pinching singularity ${ }^{14}$. First, the singularity is localised, causing break-up at a specific point on the jet free surface. Second, for low-viscosity fluids, self-similar thinning is highly asymmetric, with the uniform filament region joining to a steep main drop via a connecting neck region at each end of the filament. In a symmetric flow, break-up occurs at both necks simultaneously and the filament region contracts to form a smaller satellite drop, the mechanism of which has recently been studied by Eggers et $a l^{15}$.

However, satellites can form in several ways depending on the nature of the applied initial disturbance, as shown in the experimental work of Chaudhary et $a l^{16}$. In most liquid jet applications, including CIJ printing, it is necessary to excite the jet via a 


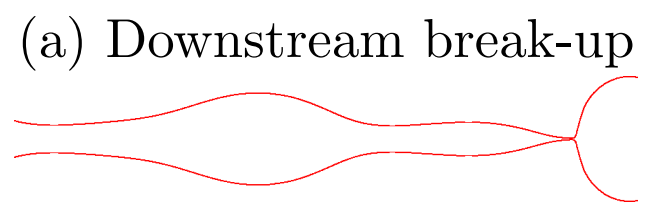

(b) Symmetric break-up

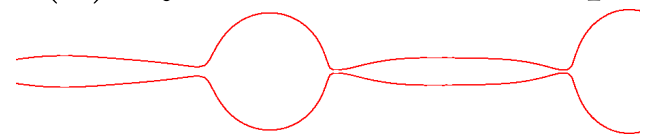

(c) 'Inverted' break-up



FIG. 1. Schematic figure showing different break-up behaviour: (a) downstream pinching; (b) symmetric pinching; (c) upstream pinching. Upstream pinching produces 'inverted' break-up that is preferable for CIJ printing.

velocity perturbation, which creates a disturbance wave that grows with distance from the nozzle exit, rather than time. Keller et $a l^{17}$ show that this disturbance is only equivalent to a temporally growing Rayleigh wave in the limit of infinite Weber number.

In light of this result, Pimbley \& Lee ${ }^{18}$ conduct a non-linear analysis of the drop formation problem via a spatial instability analysis. They find that the two most relevant parameters controlling satellite formation are the amplitude and wavelength of the initial perturbation. For certain values of these parameters, the first pinching event can occur downstream of the connecting filament, on both sides simultaneously, or upstream of the connecting filament, as shown schematically in Figure 1. In this paper, we call the phenomenon of upstream pinching 'inverted' break-up.

The theory of Pimbley \& Lee ${ }^{18}$ agrees qualitatively with the experimental findings of Chaudhary et al. ${ }^{16}$, in that there is a transition from downstream to upstream pinching for increasing perturbation amplitude and the amplitude defining the transition increases with wave number. Similar break-up patterns have also been observed in largescale CIJ experiments and industrial CIJ systems $^{6}$. Furthermore, Chaudhary et al..$^{19}$ show experimentally that this break-up behaviour can be controlled by forcing the jet with a suitable harmonic component added to the initial velocity profile. However, these studies do not explore the effect of changing Weber number, which has been shown by Vassallo et $a l .{ }^{20}$ to have a significant effect.

In order to control break-up and increase printing speed, CIJ printing exploits the effects of finite-amplitude modulations in the driving velocity profile. In particular, the driving amplitude for which inverted breakup is achieved is considered optimal for CIJ printing, since satellite drops can be eliminated by forward merging of the filament region into the leading main drop. However, this optimum operation window is not well defined. Although a full axisymmetric jet- 
ting model has been previously applied to the CIJ problem to investigate the transition from downstream to inverted break-up, with quantitative agreement to experimental data $^{6}$, the implementation of this technique is computationally expensive.

In this paper, we develop a model based on the slender-jet approximation to explore the stability and break-up of a continuous jet for a parameter range typical of CIJ printing. Despite operating at large Weber numbers, jet stability deviates from linear Rayleigh behaviour due to finite amplitudes imposed on the driving velocity, which are usually employed to encourage break-up. This causes the growth of non-linear interactions that significantly influence break-up behaviour, and is in contrast to growth of disturbances forced by perturbations of the jet diameter.

We identify the critical amplitude range for which break-up becomes inverted and present a Fourier analysis to identify the harmonic responsible for this transition. Finally, we show that inverted break-up can be controlled by the addition of a second harmonic to the driving signal. The results of our onedimensioanl (1D) model are shown to be in qualitative agreement with full axisymmetric simulations that include a simplified nozzle geometry, which is absent from the 1D model. The (full nonlinear) curvature term is given

\section{1D JETTING MODEL}

\section{A. Governing Equations}

A simplification to the full NavierStokes equations is to assume a slender-jet approximation $^{21}$ where the wavelength is assumed to be sufficiently long that the jet velocity and stress are independent of the crosssectional area, but retain the nonlinear terms in the surface curvature.

The kinematics of motion are therefore approximated as one-dimensional, depending only on the axial $z$-direction and time $t$. Thus, this 1D approximation assumes a plugflow velocity profile. Although flow through a nozzle generates a Poiseuille flow, it has been shown that for a sufficient distance downstream of the nozzle exit, the figure velocity profile of a continuous jet relaxes to a plug flow $^{22}$. Hence, a 1D model is expected to be sufficient to model the downstream dynamics of a continuous inkjet.

Denoting jet radius $h(z, t)$ and velocity $v(z, t)$, conservation of mass and momentum are given respectively by

$$
\begin{aligned}
\frac{\partial h^{2}}{\partial t}+\frac{\partial}{\partial z}\left(h^{2} v\right) & =0 \\
\frac{\partial}{\partial t}\left(h^{2} v\right)+\frac{\partial}{\partial z}\left(h^{2} v^{2}\right) & =\frac{\partial}{\partial z}\left(h^{2}\left(K+3 O h \frac{\partial v}{\partial z}\right)\right)
\end{aligned}
$$


by

$$
K \equiv \frac{1}{h\left(1+h_{z}^{2}\right)^{1 / 2}}+\frac{h_{z z}}{\left(1+h_{z}^{2}\right)^{3 / 2}},
$$

where the subscript denotes differentiation with respect to $z$ and the dimensionless Ohnesorge number is

$$
O h=\frac{\mu}{\sqrt{\rho \gamma R}}
$$

for viscosity $\mu$, density $\rho$ and surface tension $\gamma$.

The jet velocity is non-dimensionalised with respect to the nozzle radius $R$ and Rayleigh capillary time $\sqrt{\rho R^{3} / \gamma}$ so that the dimensionless velocity is defined in terms of the Weber number

$$
W e=\frac{\rho U^{2} R}{\gamma},
$$

for mean jet velocity $U$. That is, the mean initial dimensionless velocity at the nozzle exit is given by

$$
v(0,0)=v_{0}=\sqrt{W e} .
$$

The Reynolds' number is then given by

$$
R e=\frac{\sqrt{W e}}{O h} .
$$

Performing a linear stability analysis on Eqs. 1 yields the dispersion relation

$$
\alpha^{2}+3 O h k^{2} \alpha-\frac{k^{2}}{2}\left(1-k^{2}\right)=0
$$

for dimensionless growth rate $\alpha$ and wavenumber $k$. The maximum growth rate is given by

$$
\alpha^{*}=(2 \sqrt{2}+6 O h)^{-1} \text {. }
$$

Thus, in the limit of small Ohnesorge number $\alpha^{*} \approx 1 / 3$, and corresponds to wavenumber $k^{*} \approx 0.7$ or equivalently wavelength $\lambda^{*} \approx 9$, as shown in Ref. ${ }^{23}$.

The governing equations (1) are solved using a semi-implicit numerical scheme on a Eulerian grid for a range of boundary conditions chosen to replicate different driving methods, as discussed in the next section. Further details of the numerical method are given in Ref. ${ }^{23}$. We then compare our results to Rayleigh's dispersion relation (Eq. 7).

\section{B. Driving Profiles}

In the frame-work of our 1D model, the details of the nozzle geometry are neglected and we consider dynamics outside the nozzle. In order to drive an instability, we can impose two different driving profiles: either a perturbation of the cross-sectional area at the nozzle exit, or a perturbation of the velocity profile at the nozzle exit.

Perturbations to the cross-sectional area mimic thermal fluctuations in the nozzle ${ }^{1}$, and a similar approach has been taken by van Hoeve et al. ${ }^{7}$. In our model, the crosssectional area is perturbed at the nozzle exit $(z=0)$ via

$$
h^{2}(0, t)=(1+\epsilon \sin (2 \pi f t)),
$$

to induce a free-surface perturbation. Here $\epsilon$ is the driving amplitude and $f$ is the driving 
frequency. In this case, the velocity profile is constant (unperturbed) at the nozzle exit, such that

$$
v(0, t)=v_{0}
$$

where $v_{0}$ is given by Eq. 5 .

For small amplitudes $(\epsilon \leq 0.01)$, a (sinusoidal) Rayleigh instability wave is propagated downstream from the nozzle exit, provided that the Weber number is sufficiently large ${ }^{17}$. The jetting frequency is defined as

$$
f=\frac{\sqrt{W e}}{\lambda}
$$

for dimensionless wavelength $\lambda$. By choosing $\lambda=\lambda^{*} \approx 9$, the fastest growing disturbance dominates the flow, in the limit of small amplitude disturbances and low viscosity.

On the other hand, perturbations in the velocity profile mimic jets driven by a pressure modulation. In our model the velocity profile is perturbed at the nozzle exit via

$$
v(0, t)=v_{0}(1+\epsilon \sin (2 \pi f t)),
$$

where $v_{0}$ is given by Eq. 5 and the jet radius fixed at

$$
h(0, t)=1 .
$$

Perturbations of the velocity profile do not necessarily translate to a sinusoidal variation in the free-surface height and so the instability is not necessarily related to a typical Rayleigh wave. Furthermore, industrial CIJ printers also typically operate at large modulation amplitudes $(\epsilon>0.01)$ meaning that non-linear interactions are important.

\section{Break-up Criterion}

We define a break-up criterion to be when $h$ becomes less than a cut-off radius $h_{c}$, which we typically set at $1 \%$ of the nozzle radius. When $h=h_{c}$, the fluid ahead of the break point is separated from the remaining jet and evolves to form a drop. However, since the slender-jet approximation ceases to be valid, the shape of the drop is not well described and so separated drops are removed from the simulation. The distance from the nozzle exit to the point of break-up is defined to be the the break-up length, $L$.

\section{RESULTS}

In this section, we illustrate the jetting and break-up behaviour of a velocity-driven continuous jet (i.e. Eq.12). Equivalent predictions for an instability induced by a surface-perturbation (i.e. Eq.9) are deferred to the discussion. Model parameters are set to $W e=338, O h=0.122, \lambda=9$.

Fig.2 shows the dimensionless break-up length $L$ achieved in a velocity-driven jet for a range of amplitudes $\epsilon$. Since the amplitude of a linear instability increases exponentially 


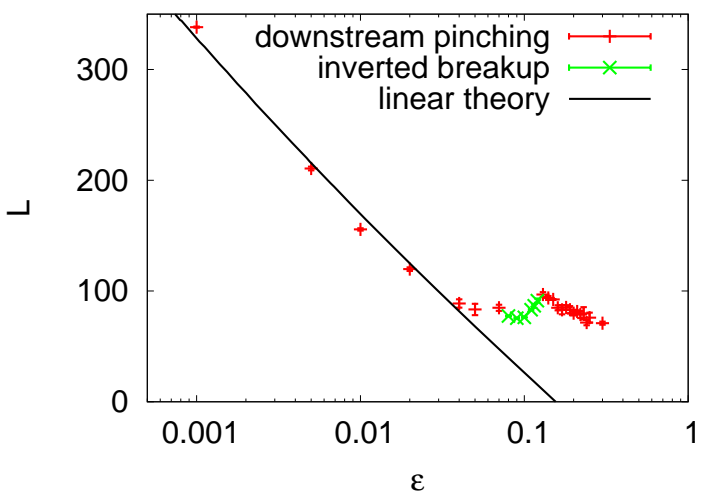

FIG. 2. Dimensionless break-up length $L$ of a velocity-driven jet (Eq.12) for a range of modulation amplitudes $\epsilon$. Inverted break-up behaviour is indicated in green. The model parameters are $W e=338, O h=0.122$ and $\lambda=9$.

with downstream distance, the break-up length decreases logarithmically with driving amplitude for small values. In particular, for sufficiently small amplitudes, the model results are in quantitative agreement with the linear theory of Garcia et $a ._{.}{ }^{4}$, which derives a simple transfer function using dispersion relation (Eq.7) to predict the break-up length of a pressure-modulated capillary jet. However, once the driving amplitude exceeds $\epsilon=0.015$, we observe a deviation from this logarithmic decay, indicating that non-linear interactions contribute to the jetting dynamics.

Fig.3 shows the free-surface profiles predicted by the 1D model for amplitudes $\epsilon=$ $0.05,0.1,0.15$. As predicted by the theory of Pimbley \& Lee ${ }^{18}$, the position of the first pinch-off event, within a single jet wavelength, is very much dependent on the driving amplitude.

For $\epsilon=0.05$, rather than a sinusoidal instability wave, we observe the development of non-linear bulges on the free surface that eventually form main droplets. The connecting filament thins, and the break position is located on the front side of the connecting filament, so that pinching occurs downstream of the main drop. Increasing the amplitude to $\epsilon=0.1$ causes the bulging to become more pronounced and, with sufficiently large non-linear interactions, inverted break-up is achieved. A similar transition from downstream to upstream break-up has been observed in large-scale CIJ experiments and industrial CIJ systems ${ }^{6}$. However, for $\epsilon=0.15$ the break-up behaviour predicted by the one-dimensional model reverts back to downstream pinching, despite larger nonlinear interactions, suggesting that a stabilising mechanism is introduced at very large amplitudes.

Evidently, there exists a critical window in the modulation amplitude that generates the desired break-up behaviour. In this case, we find that the optimum operation window for CIJ printing is restricted to $0.08 \leq$ $\epsilon \leq 0.12$. This operation window is highlighted in Fig.2 where break-up length is plotted against modulation amplitude; the 
(a) $\varepsilon=0.05$

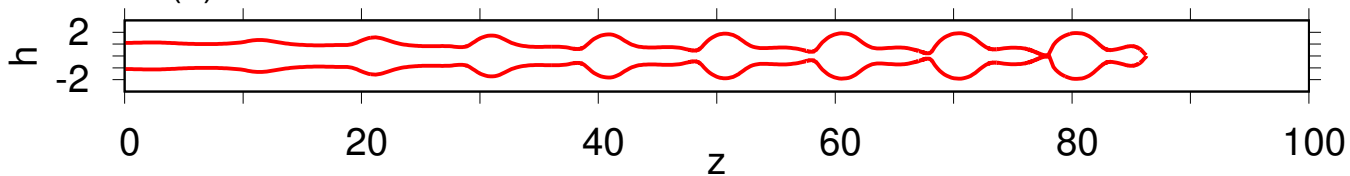

(b) $\varepsilon=0.10$

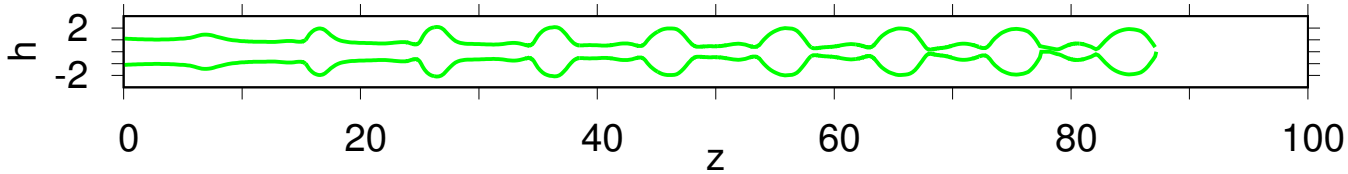

(c) $\varepsilon=0.15$

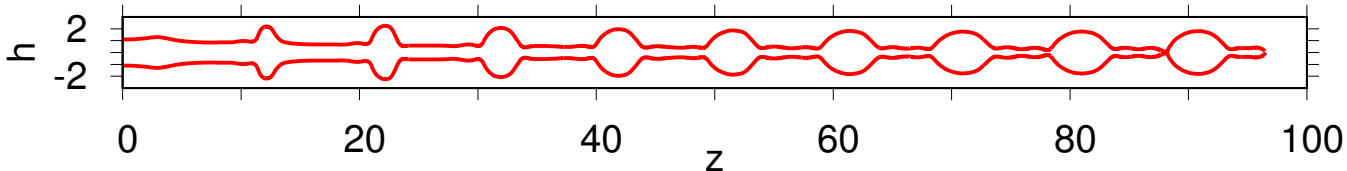

FIG. 3. Velocity-driven free-surface profiles predicted by 1D model with driving amplitudes: (a) $\epsilon=0.05$; (b) $\epsilon=0.1 ;$ (c) $\epsilon=0.15$. The model parameters are $W e=338, O h=0.122$ and $\lambda=9$.

region of inverted break-up is indicated by the green ' $\mathrm{x}$ ' data points, whereas red ' + data points correspond to downstream pinching. In the region of inversion, the breakup length increases with driving amplitude, a phenomenon also observed in the data generated by industrial jetting systems ${ }^{25,26}$. However, when the break-up behaviour reverts back to the downstream position, the breakup length again decreases with amplitude.

Moreover, this window of preferable break-up is sensitive to a number of parameters, including Ohnesorge number, Weber number and wavelength ${ }^{23}$. In particular, increasing the Ohnesorge number shifts the inverted regime to a higher modulation range. Since larger Ohnesorge numbers are stabilising, it is more difficult to drive the jet forward and therefore larger driving amplitudes are required to induce the non-linear interactions required for the break-up to become inverted. For sufficiently large Ohnesorge numbers, inverted break-up is not achieved. In contrast, increasing the Weber number makes it easier to drive the jet forward and so the inverted regime occurs at lower driving amplitudes. For sufficiently small Weber numbers, inverted break-up is not achieved. Furthermore, in agreement with the theory by Pimbley \& Lee ${ }^{18}$, the inverted break-up regime also depends on the wavelength of the initial disturbance and is shifted to a larger range of amplitudes by decreasing the wavelength. 
(a) Free-surface-driven jet



(b) Velocity-driven jet

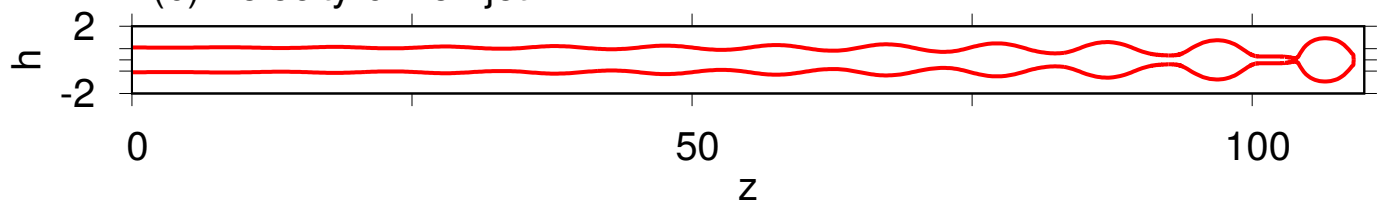

FIG. 4. Free-surface profiles predicted by 1D model for driving methods: (a) perturbing the free surface (Eq.9) and, (b) perturbing the velocity (Eq.12) at the nozzle exit. The model parameters are $W e=81, O h=0.122, \lambda=9$ and $\epsilon=0.01$.

\section{DISCUSSION}

\section{A. Linear $(\epsilon \leq 0.01)$ behaviour}

In this section, we compare the results of our 1D jetting model with Rayleigh's linear stability theory (Eq.7) for driving methods given by Eqs.9 and 12, respectively, with small driving amplitudes. Model parameters are chosen to be $W e=81, O h=0.122, \lambda=9$ and $\epsilon=0.01$.

Fig.4 shows that the free-surface profiles predicted for each driving method are almost identical. In this case, a small perturbation in the driving velocity profile induces a sinusoidal-like variation in the jet radius (Fig.4(b)), producing a profile similar to the free-surface-driven jet shown in Fig.4(a); pinching occurs downstream of the connecting filament for both driving methods. Similar downstream break-up behaviour has been observed experimentally and compared to a one-dimensional model by van Hoeve et al. ${ }^{7}$.

The main difference between the two driving methods is that driving the velocity profile generates a significantly shorter jet compared to driving the free-surface at the nozzle exit. It has been shown that the break-up length $L$ of the fastest growing mode is given by $^{11}$

$$
L=A \alpha^{*} \sqrt{W e},
$$

where $\alpha^{*}$ is the growth rate of the fastest mode (Eq. 8). The prefactor $A$ is confirmed to depend on the initial disturbance, as demonstrated here, and is usually determined experimentally. (Note that the model results have been shown to be in agreement with Eq. 14 for increasing Weber number in other work ${ }^{23}$.)

In this case, we find that the break-up length of the velocity-driven jet is half that 
(a) free-surface driven



(b) velocity driven

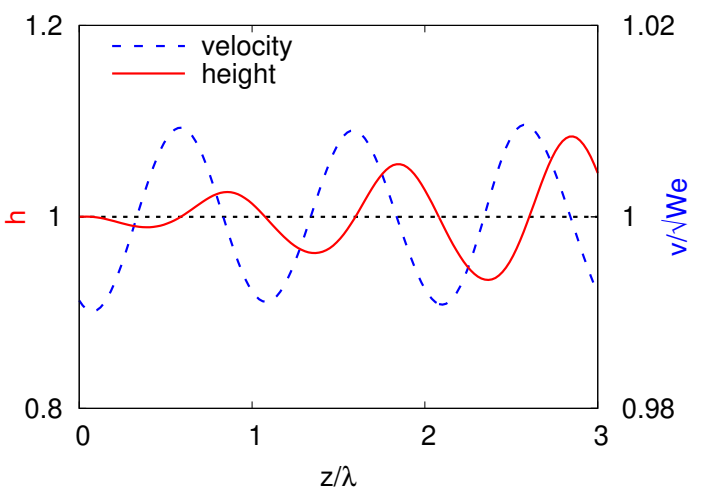

FIG. 5. Free-surface height and velocity profiles near to the nozzle exit for (a) a free-surface driven jet (Eq.9) and (b) a velocity-driven jet (Eq.12). Driving the velocity profile results in a larger surface disturbance and consequently a shorter break-up length. The model parameters are $W e=81, O h=0.122, \lambda=9$ and $\epsilon=0.01$.

of the surface-driven jet (Fig.4). Thus, the prefactor $A$ in Eq. 14 differs by a factor $\sim 2$ depending on the driving mechanisms. In fact, the predicted break-up length of the velocity-driven jet is equal to that obtained with a cross-sectional area perturbation of amplitude $\epsilon \sim 0.15$.

From linear stability analysis of the fastest growing mode, the prefactor $A$ is determined by

$$
A=\ln \left(\frac{\bar{h}}{h^{\prime}}\right)
$$

where $\bar{h}=1$ is the mean jet radius, and $h^{\prime}$ is the amplitude of the disturbance wave. Furthermore, for a velocity perturbation of amplitude $v^{\prime}$, it is found that the amplitude of the resulting free-surface disturbance is given by

$$
\left|h^{\prime}\right|=\frac{\pi}{\alpha^{*} \lambda^{*}} v^{\prime}
$$

(For full details see Ref. ${ }^{23}$.)

In this case, driving the velocity at $z=$ 0 with amplitude $\epsilon=0.01$ yields a velocity perturbation of the size $v^{\prime}=\epsilon \sqrt{W e}=0.09$. Thus, by Eq.16 it is expected to induce a freesurface disturbance of size $h^{\prime} \approx 0.1$ (for $O h=$ 0.122 and $\left.\lambda^{*} \approx 9\right)$. This is equivalent to a cross-sectional-area disturbance of size $h^{\prime 2} \approx$ 0.13. On the other hand, driving the crosssectional area with amplitude $\epsilon=0.01$ yields a smaller disturbance of $h^{\prime} \approx 0.005$ (since $h^{\prime}=\sqrt{1+\epsilon}-1$ from Eq. 9). Consequently by Eq.15, the prefactor $A$ and the resulting break-up length $L$ differ for the two driving mechanisms by approximately a factor of 2 .

To test this theory, Fig. 5 contrasts the free-surface height and velocity profiles found near to the nozzle exit (i.e. within the first three wavelengths of the disturbance wave) for each driving method with $\epsilon=0.01$. We find that a velocity perturbation $v^{\prime}=\epsilon \sqrt{W e}$ 
induces a smaller free-surface disturbance than predicted by Eq.16 in the vicinity of the nozzle. However, this disturbance increases rapidly with distance from the nozzle, resulting in the shorter break-up length we find.

Nevertheless, the variation in break-up length $L$ with wavelength $\lambda$ is the same for both driving methods and can be predicted from the growth rates obtained from the Rayleigh dispersion relation (Eq.7), as shown in Fig. 6.

For both driving methods, we find that the shortest break-up length is generated by the most unstable wavelength $\lambda^{*} \approx 9$ and that longer jets can be generated by imposing a different frequency due to the decreased growth rate of the disturbance wave. We observe a small discrepancy from the dispersion relation when driving the velocity component, since an exact sinusoidal height profile is not achieved. However, it is clear that the jet stability is dominated by linear dynamics in this parameter range for both driving methods. We now consider the effect of increasing the perturbation amplitude.

\section{B. Non-linear $(\epsilon>0.01)$ behaviour}

As shown earlier (Fig.3), large-amplitude velocity perturbations can significantly alter the break-up profile, as well as the break-up length. In particular, Fig. 7 compares the

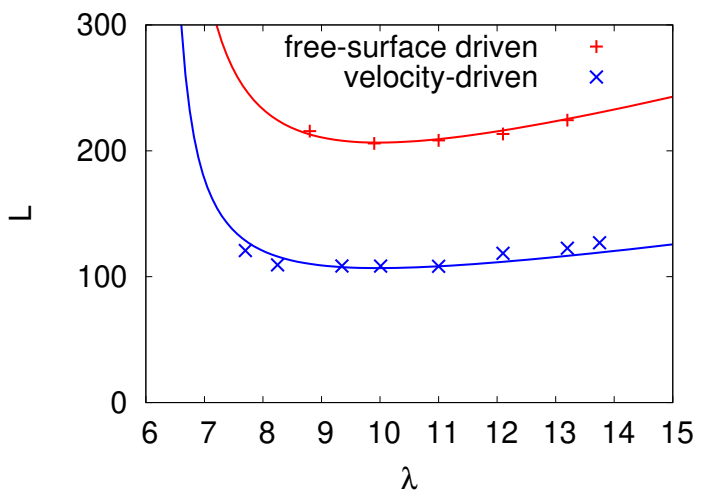

FIG. 6. Break-up length $L$ predicted by freesurface (9) and velocity (12) driving for a range of wavelengths $\lambda$ compared to the inverse of Rayleigh's dispersion relation (7). The model parameters are $W e=81, O h=0.122$ and $\epsilon=0.01$

free-surface profile predicted for each of the two driving methods Eq.9 and Eq.12 with $\epsilon=$ 0.1. Although applying a large perturbation to the cross-sectional area to drive break-up (Eq.9) demonstrates the same downstream pinching we observed in the previous section, the pinch position is inverted to upstream when break-up is driven by modulations of the velocity profile.

In order to highlight this non-linear behaviour, Fig.8 shows how the predicted break-up length of a velocity-driven jet with $\epsilon=0.05$ diverges from the inverse of Rayleigh's dispersion relation (Eq.7); breakup length increases with wavelength, rather than obeying the linear theory as seen for smaller driving amplitudes (Fig.6). More- 
(a) Free-surface-driven jet



(b) Velocity-driven jet

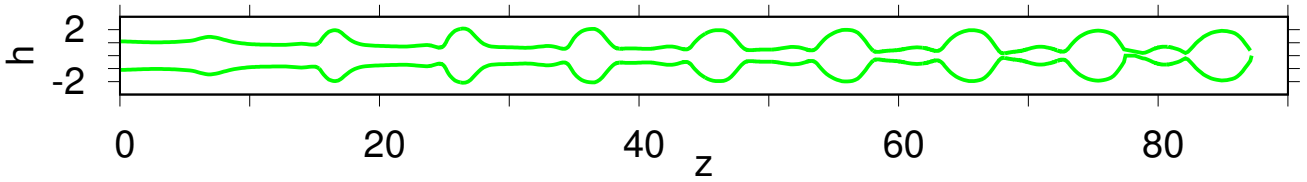

FIG. 7. Free-surface profile predicted by the one-dimensional model for driving methods: (a) modulating the cross-sectional area at the nozzle exit given by Eq.9; (b) modulating the driving velocity as given by Eq.12. The model parameters are $W e=338, O h=0.122, \lambda=9$ and $\epsilon=0.1$.
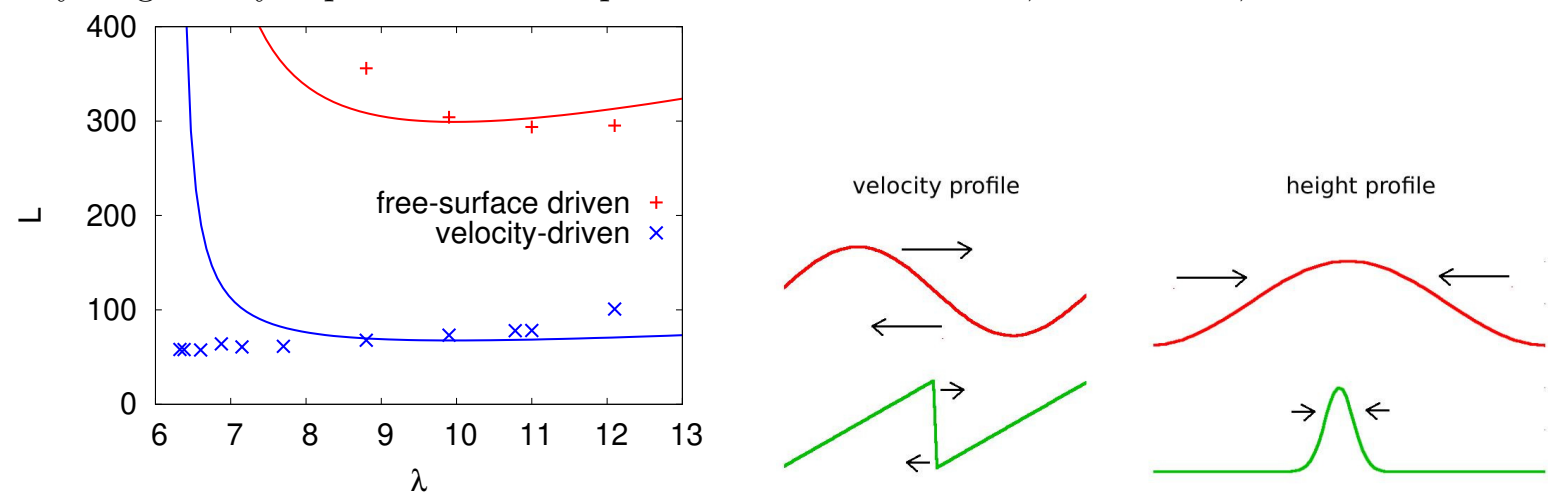

FIG. 8. Break-up length $L$ of a velocity-driven FIG. 9. Schematic figure showing how the veloc(Eq.12) for a range of wavelengths $\lambda$ compared ity profile evolves into a sawtooth wave causing to the inverse of Rayleigh's dispersion relation isolated bulges to form on the uniform thread.

(Eq.7). The model parameters are $W e=338$,

$O h=0.122$ and $\epsilon=0.05$.

over, the fastest growing disturbance wave is found to have wavelengths smaller than the classic Rayleigh wave $\left(\lambda^{*} \approx 7\right.$, rather than $\lambda^{*} \approx 9$ ). This non-linear effect has also been observed in experiments ${ }^{27}$.

It is also worth noting that if we apply enough forcing this non-linear jet does not appear to stabilise at $\lambda \approx 2 \pi$. Furthermore, the model predicts a similar deviation from the linear theory for larger amplitudes $\epsilon=0.1$ and 0.15 (not shown). Finally, the stability of a surface-driven jet also deviates from Rayleigh's theory. However in contrast to the the velocity-driven instability the fastest growing disturbance wave shifts to slightly larger wavelength $\lambda^{*} \approx 10$ (Fig.8). 
At this large amplitude, the surface variation produced by modulating the driving velocity is not similar to a sinusoidal wave; the jet velocity is distorted due to the non-linear advection term appearing in conservation on momentum:

$$
\left(v-\frac{6 O h}{h} \frac{\partial h}{\partial z}\right) \frac{\partial v}{\partial z}
$$

Due to this non-linearity, the peak of the velocity profile travels faster than the trough, so that the pulse becomes accumulatively more like a sawtooth wave and generates a 'shock' in the velocity profile, as sketched in Fig.9.

Consequently, fluid upstream of the shock moves faster than the fluid downstream causing steep bulges to form on the uniform thread. This behaviour is evident is the freesurface predicted by the 1D model shown in Fig.7(b). Due to this distortion, other frequency components are introduced, which we investigate later via a Fourier analysis, and it is possible to invert the pinching from the downstream position to upstream for sufficiently strong non-linear interactions. However, the generation of this preferable inverted break-up behaviour in velocity-driven jets is restricted to a narrow operating window, as discussed earlier (Fig.2).

\section{Full axisymmetric simulations}

Full axisymmetric simulations were performed using the method of Harlen et $a t^{28}$ to validate the $1 \mathrm{D}$ model. The code uses a Eulerian-Lagrangian finite-element $\operatorname{method}^{29}$ to capture the evolving free-surface shape and has previously been used to study jet break-up in drop-on-demand printing for both Newtonian ${ }^{30}$ and viscoelastic fluids ${ }^{28}$ and CIJ printing of Newtonian fluids ${ }^{6}$.

The software uses a moving-mesh, finiteelement method to solve the Navier-Stokes equation

$$
\rho\left(\frac{\partial \mathbf{u}}{\partial t}+\mathbf{u}(\nabla \cdot \mathbf{u})\right)=-\nabla p+\nabla \cdot \boldsymbol{\sigma}
$$

for axisymmetric jet velocity $\mathbf{u}=\left(u_{r}, 0, u_{z}\right)$, pressure $p$ and stress tensor $\boldsymbol{\sigma}$ along with the incompressibility condition

$$
\nabla \cdot \mathbf{u}=0
$$

By allowing the finite elements to deform with the fluid velocity, the Newtonian con-



FIG. 10. Initial grid used in CIJ simulations for nozzle aspect ratio 1 .The simplified print head geometry is assumed to be axisymmetric. 


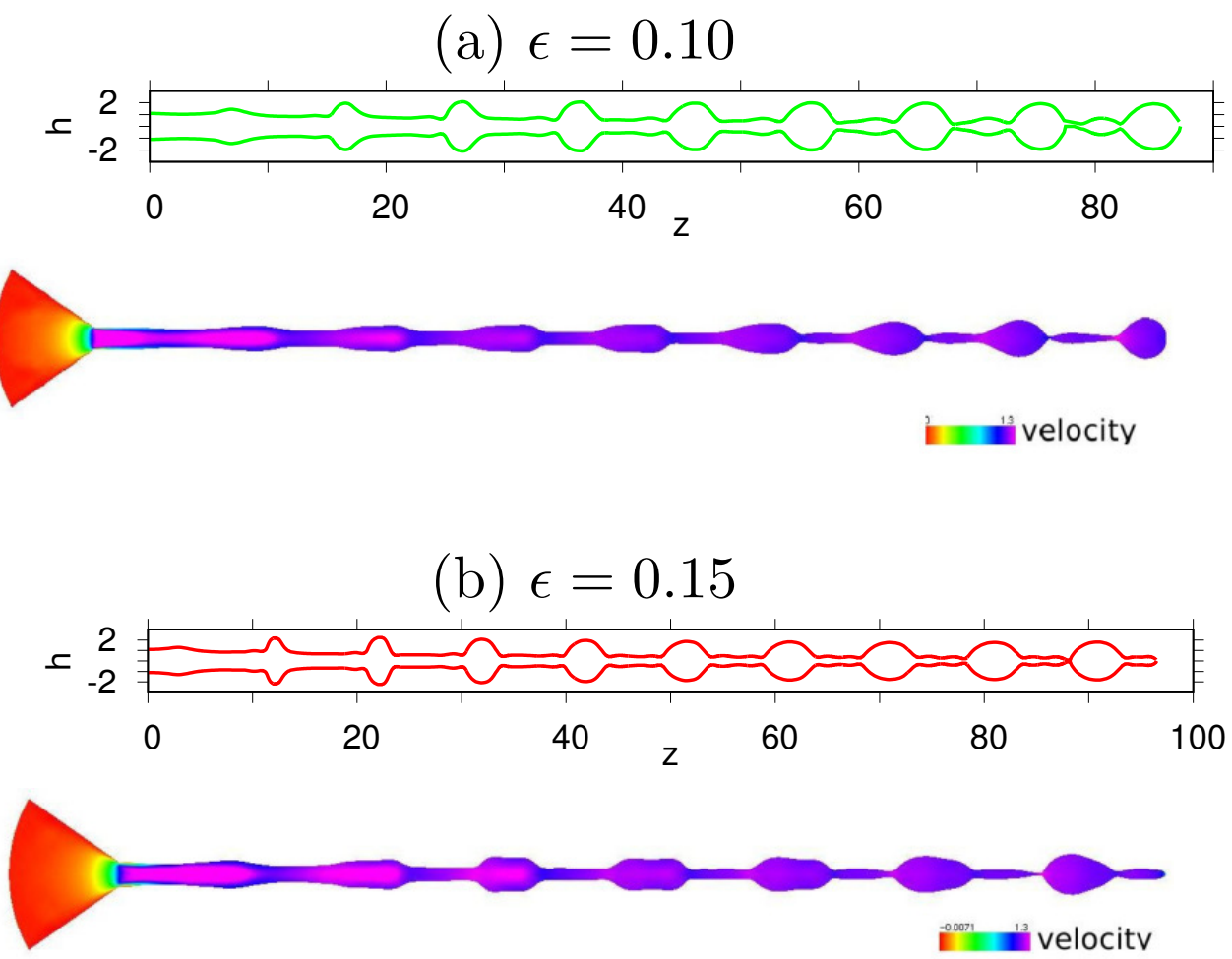

FIG. 11. Free-surface profile predicted by the one-dimensional CIJ model compared to the full axisymmetric simulation with nozzle aspect ratio $\Gamma=1$ for $(\mathrm{a}) \epsilon=0.1$ and $(\mathrm{b}) \epsilon=0.15$. The parameters are $W e=338, O h=0.122, \epsilon=0.15$ and $\lambda=9$. The color bar indicates the crosssectional jet velocity in the axisymmetric case. The one-dimensional and full simulation results are plotted with the same aspect ratio and length scale.

stitutive equation

$$
\boldsymbol{\sigma}=\mu\left(\boldsymbol{K}+\boldsymbol{K}^{T}\right)
$$

is solved in the co-deforming frame for viscosity $\mu$ and velocity gradient tensor $K_{i j}=\frac{\partial u_{i}}{\partial x_{j}}$. At the fluid-air interface the boundary condition is defined to be

$$
[\boldsymbol{\sigma} \cdot \hat{\mathbf{n}}]_{a i r}^{i n k}=-\gamma\left(\frac{1}{R_{1}}+\frac{1}{R_{2}}\right) \hat{\mathbf{n}},
$$

where $\hat{\mathbf{n}}$ is the unit vector normal to the of the actual print head behind the nozzle interface, $\gamma$ is surface tension and $R_{1}, R_{2}$ by assuming axisymmetry (real print heads 
are typically non-axisymmetry). The initial finite-element grid, with a nozzle aspect ratio 1, is shown in Fig. 10 and has previously been described by Casterjon-Pita et al. ${ }^{6}$. For an unmodulated jet with mean velocity $U$ at the nozzle outlet, the magnitude of the velocity applied at the inlet is

$$
u_{0}=\frac{\pi R^{2} U}{A_{\text {in }}},
$$

where $R$ is the nozzle radius and $A_{\text {in }}$ is the surface area of the print head inlet surface. To simulate a jet with modulation of frequency $f$ and amplitude $\epsilon$, the inlet velocity is prescribed in terms of time $t$ as

$$
u_{\text {noz }}(t)=u_{0}(1+\epsilon \sin (2 \pi f t)) .
$$

Again, while this is not expected to fully represent the experimental flow throughout the entire print head, it is designed to produce sufficiently similar flow conditions in the vicinity of the nozzle. The possible presence of higher harmonics in the jet actuation is neglected and no method of coalescence is implemented in the simulations.

Fig. 11 compares the free-surface profiles predicted by the $1 \mathrm{D}$ model to that predicted by the full axisymmetric simulation. Typical CIJ parameters are chosen to be $O h=0.122$ and $W e=338$; the frequency is determined by the Rayleigh wavelength $\lambda^{*} \approx 9$ and the amplitude of the modulation is set to $\epsilon=0.1$ and $\epsilon=0.15$, respectively. The nozzle aspect ratio of the full simulation, defined by

$$
\Gamma=\frac{L_{n o z}}{R},
$$

for nozzle length $L_{n o z}$, is set to $\Gamma=1$.

The colour scale red-to-pink, shown for the full simulation, indicates minimum-tomaximum jet velocity. The Poiseuille flow generated by the simplified nozzle geometry in the full simulation has a stabilising influence on the jet, increasing the breakup length compared to the one-dimensional model, and also elongates the shape of the main drops. However, qualitatively the break-up behaviour of the two models are in agreement for both driving amplitudes; for $\epsilon=0.1$ we observe the preferable inverted break-up, whereas for $\epsilon=0.15$, pinching reverts to the downstream position.

\section{A secondary instability wave}

As first suggested by Pimbley \& Lee ${ }^{18}$, large modulations in the driving velocity induce non-linear interactions that generate a secondary instability wave. Indeed, if a component with a shorter wavelength has the chance to grow sufficiently with respect to the fundamental, it will produce an additional growth of surface perturbations across the connecting filament and cause inverted pinching, as suggested in Ref. ${ }^{21}$.

To understand this secondary instability wave, we decompose the free-surface profile 


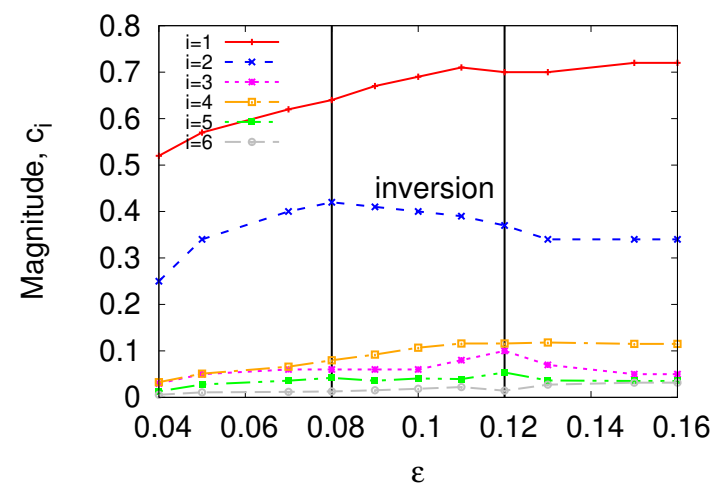

FIG. 12. Magnitude of Fourier coefficients $c_{i}$ at $z=50$ plotted as a function modulation amplitude $\epsilon$.

into its Fourier components. In this way, the jet is divided into a series of simpler functions and we are able to identify the importance of the second harmonic, relative to the fundamental mode, at amplitudes that give inverted break-up.

The time-dependent height at $z=50$, where the pinch location is established, is approximated by a Fourier series of the form $h_{50}(t)=a_{0}+\sum_{i=1}^{5} a_{i} \cos (2 \pi i f t)+b_{i} \sin (2 \pi i f t)$,

where $f$ is the Rayleigh frequency. The magnitude of the Fourier coefficients

$$
c_{i}=\sqrt{a_{i}^{2}+b_{i}^{2}}, \quad i=1, \ldots 5,
$$

enables the magnitude of the secondary harmonic to be compared over a range of amplitudes.

In particular, Fig.12 suggests that the first four Fourier components are the most im- portant, as suggested by the second-order, non-linear analysis given by Chaudhary et $a l .{ }^{19}$. The fundamental solution is clearly the largest and increases as jet modulation is increased. The second harmonic is the secondlargest component, although the relative size depends on the modulation amplitude. The third, fifth and sixth harmonics remain small, whereas the fourth harmonic although small, grows with increasing amplitude.

In the region of inverted break-up $(0.08 \leq$ $\epsilon \leq 0.12$ ), the magnitude of the second harmonic is shown to be significantly larger than in the regions of downstream pinching (Fig. 12). Thus, the growth of a secondary instability wave causes a transition at $\epsilon=0.08$ from downstream pinching to inverted breakup, in agreement with the theory of Pimbley $\&$ Lee $^{18}$. The transition at $\epsilon=0.12$ from inverted break-up back to downstream pinching can be explained as follows.

At this large amplitude the filament region becomes 'frustrated' - a term used by Pimbley $\mathrm{Lee}^{18}$ to describe the reduction in growth rate at higher amplitudes. This behaviour is due to the growth of higher-order harmonics that are stable to the Rayleigh instability, since $k_{i} h_{f}>1$, where $k_{i}$ is the wave number of the $i$ th-order harmonic and $h_{f}$ is the filament radius. In particular, Fig.12 shows how the magnitude of the fourth-order harmonic $\left(k_{4}=2.8\right)$ increases with modulation ampli- 


\section{(a) Original Case}

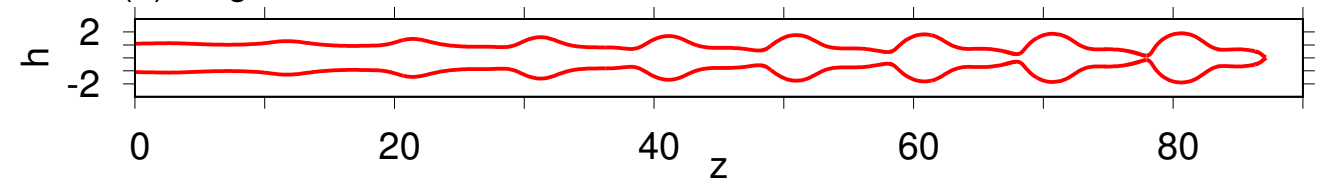

(b) Added Second Harmonic

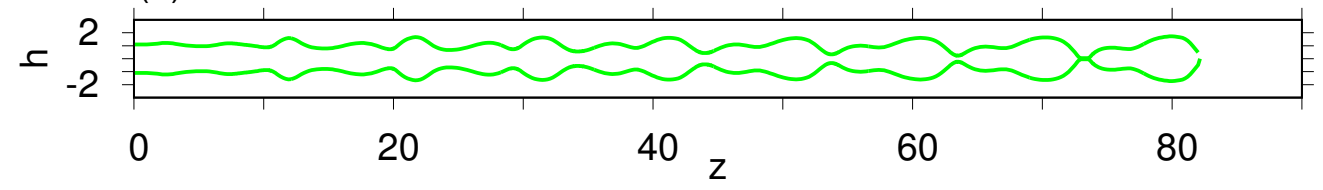

FIG. 13. Free-surface profile predicted by 1D model for (a) original driving profile (Eq.12) with $\epsilon=0.04$, and (b) second harmonic added to the driving profile (26) with $\epsilon=0.04$ and $\theta=\pi / 4$. The model parameters are $W e=338, O h=0.122, \lambda^{*}=9$.

tude, becoming relatively large for $\epsilon>0.12$. by $\theta$ and its amplitude is equal to that of Moreover, for the case $\epsilon=0.15$ the filament the fundamental $\epsilon$, as in the work of Chaudradius is $h_{f} \approx 0.5$ (Fig.3). Thus, the sta- hary et $a l .{ }^{19}$. We observe a distinct change bility criterion $k_{4} h_{f}>1$ is satisfied, causing from the usual downstream pinching, generbreak-up to occur downstream of the filament ated by the fundamental driving profile, to region. inverted break-up behaviour when forcing the

The addition of harmonics to the initial velocity profile can have a significant effect on the break-up behaviour of a continuous inkjet. In particular, Chaudhary et $a l^{19}$ have shown theoretically that the formation of satellites can be controlled by forcing the jet with a suitable harmonic added to the fundamental.

jet in this way (Eq.26), as shown in Fig.13 for driving amplitude $\epsilon=0.04$ and phase shift $\theta=\pi / 4$.

This phase shift is crucial to achieving this transition $^{23}$, although the reason remains unclear. We choose $\theta=\pi / 4$ to coincide with the phase angle of the second harmonic i.e.

As an example, we examine the effect of adding a secondary harmonic to the driving velocity profile such that

$$
\theta_{i}=\tan ^{-1}\left(\frac{b_{i}}{a_{i}}\right) \text { for } i=2
$$

$$
\begin{aligned}
v(0, t)=v_{0}(1 & +\epsilon \sin (2 \pi f t) \\
& +\epsilon \sin (4 \pi f t+\theta)) .
\end{aligned}
$$

The phase of this second harmonic is given fundamental solution (see Fig.14). 


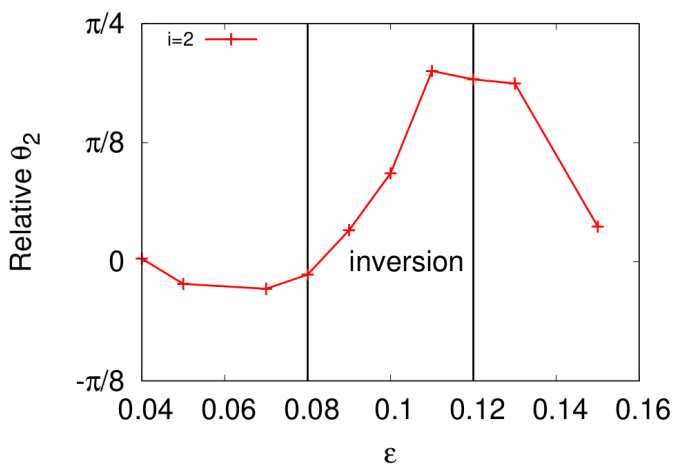

FIG. 14. Phase angle $\theta_{i}=\tan ^{-1}\left(b_{i} / a_{i}\right)$ of the second harmonic component $i=2$ calculated at $z=50$, plotted as a function modulation amplitude $\epsilon$.

\section{CONCLUSION}

We have developed a one-dimensional model to explore the break-up behaviour of a liquid jet. Driving the jet via a velocity perturbation, rather than a surface perturbation, has important consequences for the evolution of the free surface shape; for sufficiently larger amplitudes non-linear interactions can cause pinching to be inverted from the downstream to the inverted position. Furthermore, in this non-linear regime, we find that the the most unstable jets are generated at frequencies larger than the classic Rayleigh mode.

We find that preferable upstream breakup behaviour is restricted to a narrow operation window. Due to a significant reduction in computation time, compared to full axisymmetric simulations, we have been able to extend the parameter space considered in previous studies, and show that this narrow operation window is sensitive to frequency, driving speed and viscosity. Finally, a Fourier analysis has demonstrated that the growth of a second harmonic is responsible for inverted break-up, so that addition of a secondary component to the driving signal can artificially induce inverted break-up at smaller driving amplitudes.

\section{ACKNOWLEDGEMENTS}

This work was supported by the UK Engineering and Physical Sciences Research (EPSRC) through grant number EP/H018913/1 Innovation in Industrial Ink-jet Technology. The authors would like to thank Dr John Morgan and Dr Neil Morrison, and Dr Rafa Casterjon-Pita, Queen Mary University College London for helpful discussions and an enjoyable collaboration.

\section{REFERENCES}

${ }^{1}$ I. M. Hutchings and G. D. Martin, Inkjet technology for digital fabrication (John Wiley and Sons Ltd, 2013).

${ }^{2}$ Y. Liu and B. Derby, "Experimental study of the parameters for stable drop-ondemand inkjet performance," Physics of Fluids 31, 032004 (2019). 
${ }^{3} \mathrm{R}$. Savart, "Memoire sur la constitution des veines liquides lancees par orifices circulaires en mince parol," Ann. Chim. 53 (1833).

${ }^{4}$ J. H. Hilbing and S. D. Heister, "Droplet size control in liquid jet break-up," Phys. Fluids 8 (1996).

${ }^{5}$ R. P. Grant and S. Middleman, "Newtonian jet stability," AIChE J. 12, 669-678 (1966).

${ }^{6}$ J. R. Castrejon-Pita, N. F. Morrison, O. G. Harlen, G. D. Martin, and I. M. Hutchings, "Experiments and Lagrangian simulations on the formation of droplets in continuous mode," Phys. Rev. E 83 (2011).

${ }^{7}$ W. van Hoeve, S. Gekle, J. H. Snoeijer, M. Versluis, M. P. Brenner, and D. Lohse, "Break-up of diminutive Rayleigh jets," Phys. Fluids 22 (2010).

${ }^{8}$ A. Montessori, M. Lauricella, E. Stolovicki, D. A. Weitz, and S. Succi, "Jetting to dripping transition: Critical aspect ratio in step emulsifiers," Physics of Fluids 31, 021703 (2019).

${ }^{9}$ Y. Ren, K. S. Koh, J. K. Chin, J. Wang, C. Wen, and Y. Yan, "Droplet formation and fission in shear-thinning/newtonian multiphase system using bilayer bifurcating microchannel," Journal of Heat Transfer 140, 012405 (2018).

${ }^{10}$ B. W. Wagoner, S. S. Thete, and O. A. Basaran, "A new experimental method based on volume measurement for deter- mining axial scaling during breakup of drops and liquid threads," Physics of Fluids 30, 082102 (2018).

${ }^{11}$ L. Rayleigh, "On the instability of jets," Proc. of London Math. soc. 10, 4-13 (1878).

${ }^{12}$ M. C. Yuen, "Non-linear capillary instability of a liquid jet," J. Fluid Mech 33, 151163 (1968).

${ }^{13}$ H. C. Lee, "Drop formation in a liquid jet," IBM J. Res. Develop. 18, 364-369 (1974).

${ }^{14} \mathrm{~J}$. Eggers, "Universal pinching of $3 \mathrm{D}$ axisymmetric free-surface flow," Phys. Rev. Lett. 71, 3458-3460 (1993).

${ }^{15}$ J. Eggers, "Post-breakup solution of Navier-Stokes and Stokes threads," Phys. Fluids 26 (2014).

${ }^{16}$ K. C. Chaudhary and T. Maxworthy, "The non-linear capillary instability of a liquid jet. Part 2. Experiments on jet behaviour before droplet formation," J. Fluid Mech. 96, 275-286 (1980).

${ }^{17}$ J. B. Keller, S. I. Rubinow, and Y. O. Tu, "Spatial instability of a jet," Phys. Fluids 16 (1973).

${ }^{18} \mathrm{~W}$. T. Pimbley and H. C. Lee, "Satellite drop formation in a liquid jet," IBM J. Res. Develop. , 21-30 (1977).

${ }^{19}$ K. C. Chaudhary and L. G. Redekopp, "The non-linear capillary instability of a liquid jet. Part 1. Theory," J. Fluid Mech. 96, 257-274 (1980). 
${ }^{20} \mathrm{P}$. Vassallo and N. Ashgriz, "Satellite formation and merging liquid jet break-up," Proc. Royal Soc. London Ser. A 433, 269286 (1991).

${ }^{21}$ J. Eggers, "Nonlinear dynamics and breakup of free surface flows," Rev. Mod. Phys. 69 (1997).

${ }^{22}$ J. R. Castrejon-Pita, S. D. Hoath, and I. M. Hutchings, "Velocity profiles in a cylindrical liquid jet by reconstructed velocimetry," ASME J. Fluids Engineering 134 (2012).

${ }^{23}$ C. McIlroy, Complex inkjets: Particles, polymers and non-linear driving, Ph.D. thesis, Department of Applied Mathematics, University of Leeds (2014).

${ }^{24}$ F. J. Garcia, H. Gonzalez, J. R. CastrejonPita, and A. A. Castrejon-Pita, "The break-up length of harmonically stimulated capillary jets," App. Phys. Lett. 105 (2014).

${ }^{25}$ A. Kalaaji, B. Lopez, P. Attane, and A. Soucemarianadin, "Break-up length of forced liquid jets," Phys. Fluids 15 (2003).
${ }^{26}$ John Morgan, "private communication," (2014).

${ }^{27}$ G. E. A. Meier, A. Klopper, and G. Grabitz, "The influence of kinematic waves on jet break down," Experiments in Fluids 12, 173-180 (1992).

${ }^{28}$ O. G. Harlen and N. Morrison, "Viscoelasticity in inkjet printing," Rheol. Acta 49, 619-632 (2010).

${ }^{29}$ O. G. Harlen, J. M. Rallison, and P. Szabo, "A split Langrangian-Eulerian method for simulating transient viscoelastic flows," J. Non-Newt. Fluid Mech. 60, 81-104 (1995). ${ }^{30}$ J. R. Castrejon-Pita, N. F. Morrison, O. G. Harlen, G. D. Martin, and I. M. Hutchings, "Experiments and Lagrangian simulations on the formation of droplets in dropon-demand mode," Phys. Rev. E 83 (2011). ${ }^{31} \mathrm{H}$. Westborg and O. Hassager, "Creeping motion of long bubbles and drops in capillary tubes," J. Colloidal and Interface Science 133, 135-147 (1989). 